PLATE 2.

Mr. Naughton Dunn _ - - $\quad$ - Muscle and Tendon Repair
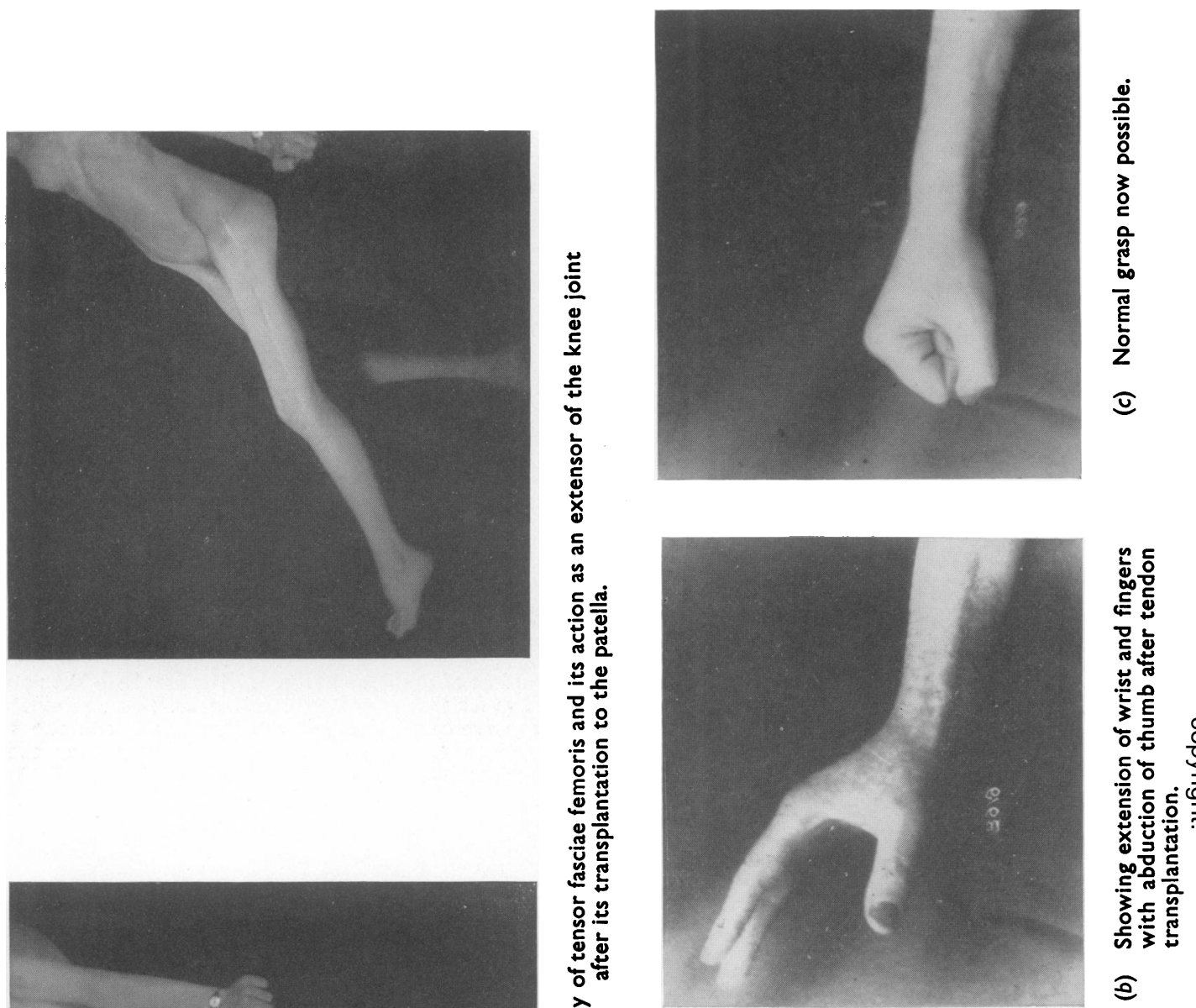

的

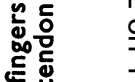

동

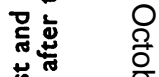

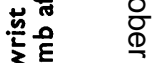

융

ᄃ힌.

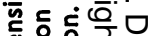

은 을 웅

×仓



उद्व

흔.

造

政造

$\widehat{\varrho}$

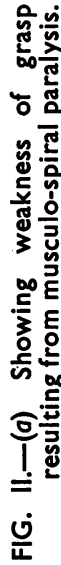

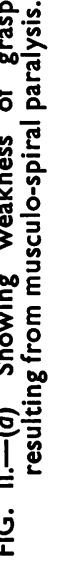




\title{
THE SURGERY OF MUSCLE AND TENDON IN RELATION TO PARALYSIS AND INJURY.
}

\author{
By NAUGHTON DUNN, LL.D., M.A., M.B. \\ (Lecturer on Orthopacdic Surgery, University of Birmingham.)
}

The treatment of muscles and tendons, whose function has been impaired as a result of disease or injury, has been a subject of surgical interest for very many years. The vast literature on this subject, published during the present century, shows that general agreement has not yet been reached as to the cause of success or failure in the many procedures which have been advised for dealing with particular problems.

I propose to devote this short paper to the principles and practice upon which from experience I personally rely. The various myopathies and progressive lesions of the central nervous system may be excluded because, although we can effect temporary improvement in function by simple division or elongation of tendons, they do not present any particular surgical problem.

Loss of function in the limbs may be due to $(a)$ lesions of the upper motor neurone, $(b)$ lesions of the lower motor neurone, $(c)$ lesions of the muscles or tendons.

\section{(a). LESIONS OF THE UPPER MOTOR NUERONE.}

This group includes the hemiplegias, paraplegias and diplegias. In these cases muscle substance is normal, but voluntary control is deficient. All muscles being in spasm, the position of the limb is determined by the pull of the stronger muscle groups. We therefore find that deformity is almost constant, and its correction depends on restoring balance by lessening the power of the stronger group. We can do this either by division of the muscles, tendons, or the nerves which supply them. Our object is to restore the joint to the position in which such voluntary power as is present may be most useful to the patient. Surgery can only be helpful in improving function, if at least a moderate degree of voluntary control is present.

The principles of treatment may be best appreciated by considering a typical case of spastic paralysis in the upper and lower extremities.

In the upper extremity the elbow is flexed, the forearm pronated, the wrist flexed, and the thumb adducted, so that such voluntary control of the digits as may be present is interfered with mainly by the spasm of the flexor group and adduction of the thumb. Prognosis in such a case will depend largely on the ability of the patient to respond to requests to carry out particular movements. If the patient is asked to supinate the forearm and in the effort to do so flexes the elbow, this is an indication that spasm of the pronator radii teres is interfering with function. Again, if, on attempting to open the hand, the wrist flexes further and the patient is unable to clear the thumb from the grasp but otherwise the effort is good, then surgical treatment will be helpful. The object of surgical treatment is to weaken the muscle groups in which tonic muscle contraction is interfering with function. The operations may necessitate division of the pronator radii teres, division of the wrist flexors, and an operation which may best be described as "filleting" the first metacarpal bone; that is, separation of all 
muscle attachments on the ulnar side of the bone, so as to prevent its adduction and interference with grasp. In more severe cases transplantation of the wrist flexors to the extensor group-which operation will be referred to later in connection with lesions of the lower neurone-or arthrodesis of the wrist may be necessary.

In upper motor neurone paralysis affecting the lower extremity the essential deformities are adduction and slight flexion of the hip, flexion of the knee, and equinus of the foot. These positions make the upright position and balance difficult or impossible. Again it is a question of weakening the stronger group of muscles. This may be effected by division of the tendons, or partial or complete resection of the nerves controlling them. In the case of the short tendo Achillis the operation of lengthening it must be regarded as a major surgical procedure. If it is divided without due care elongation of the muscle will occur and the very serious and disabling deformity of calcaneo-cavus result. The points to be remembered with regard to this most important tendon are never to divide it; if it is elongated to be sure that at the time of the operation the foot is not allowed to dorsiflex beyond the right angle; and that a position of slight equinus is maintained until true healing has occurred. The walking plaster should be worn for at least two months. These rules apply to all types of foot deformity where the tendo Achillis is short, the axiom to remember being that a slightly shortened is a far less serious disability that a slightly elongated tendo Achillis.

In some cases of spastic paralysis of the lower limbs, where voluntary control is so poor that even with surgical measures reasonable function is never likely to result, division of the adductors of the hips may be advised to facilitate nursing cleanliness.

\section{(b). LESIONS OF THE LOWER MOTOR NEURONE.}

This group consists mainly of cases of loss of power in the muscles as a result of infantile paralysis or irreparable or incomplete recovery from injuries of the peripheral nerves. The cause of deformity in these cases is different. In lesions of the upper motor neurone deformity was constant because deflection of the joints depended on tonic spasm of the stronger muscles, but in this group it results primarily from weakness of the affected muscles. Infantile paralysis differs from peripheral nerve injuries, in that in the former there is paralysis, partial or complete, of odd muscles or groups of muscles while in the latter there is complete paralysis of the definite muscle group supplied by the injured nerve. Further, as regards the surgical problem there is this difference also that in the case of peripheral nerve injuries the muscles which are available for transplantation are normal, while in infantile paralysis these, although active, may themselves be weakened.

Before discussing the operations on muscles which have proved helpful, the treatment of the deformities which result from paralysis of individual muscles or muscle groups must be considered.

Joint stability depends essentially on balanced muscular control. The action of the stronger group of muscles determines the type of deformity, while the weaker group of muscles becomes further weakened as a result of stretching and disuse. In the case of the lower extremity incipient deformity resulting from unbalanced muscular control will be aggravated by the incidence of body-weight. These deformities are preventable, so that an axiom of treatment in infantile paralysis during the acute stage and throughout the period of convalescence 
should be to maintain the weaker muscles in a position of relaxation and continue their treatment by muscle re-education and splintage until such time as we are satisfied that maximum recovery has been obtained or that deformity becomes progressive in spite of splintage. The same principles apply to muscles weakened as a result of injury of peripheral nerves.

In cases in which deformity has already occurred correction of this is necessary before successful treatment by tendon transplantation is possible. We must, however, remember that to divide a tendon weakens the power in its muscle and thus shortened muscles should be stretched by gradual splintage rather than divided. This is of special importance in cases in which the shortened muscle may be utilized by transplantation to improve function. Methods adopted for the gradual correction of deformity prior to operation have been fully described in a previous communication.*

\section{Tendon Transplantation.}

For success in tendon transplantation there is general agreement on the following principles:-

(I) That previous to operation correction of deformity should be complete.

(2) That the transplanted muscle should run in a direct line to its new insertion.

(3) That the muscle should be of sufficient power to meet the strain which will be imposed on it.

(4) A recognition of the fact that where (3) is not practicable transference of muscle power may be advantageously utilized as an adjuvant to other procedures such as tenodesis or arthrodesis.

In spite of agreement on these points, this branch of orthopædic surgery is that on which there is still much controversy. Failures have been ascribed mainly to adhesions or faulty union. Biesalski, Mayer, Steindler and Bernstein are among the main contributors to the modern literature which deals largely with the problem of the prevention of adhesions and the maintenance of nutrition of transplanted tendons. Although I appreciate their work, I do not agree with them that these are the main causes of failure in a large number of the tendon transplantations advised and practised. My experience has been that with transplantation of tendons through tunnels in the subcutaneous tissue you can be sure of success in some operations and as certain of failure in others.

On looking back on a fairly considerable experience of tendon transplantations I am satisfied that success or failure has depended mainly on two other factors-

(I) Whether the transposed tendon was one naturally in action with that whose function it was to replace or could be easily re-educated to perform its new function.

(2) The degree of tension under which the new tendon is sutured.

The movements employed in locomotion are automatic and controlled by groups of muscles so that it is difficult to re-educate a muscle to act apart from its group. I therefore regard it as an axiom that in the lower extremity a tendon should only be transplanted to replace one of its own group-i.e., one normally in action with it. In the upper extremity the action of muscles is more specialized so that re-education of an individual tendon to act apart from its group appears to be more practicable.

* The Surgery of Muscle \& Tendon in Relation to Infantile Paralysis. Proc. Roy. Soc. Med., Dec. 1928, Vol. XXII. 
These views are confirmed by my personal experience of certain operations advised in the textbooks. I would refer particularly to transplantation of the biceps to the patella in cases of quadriceps insufficiency, and transplantation of the peroneus longus muscle to replace a paralysed tibialis anticus. In both cases the tonic action and the fact that we have weakened a group that was stronger than its opponents may justify the operation as a means of increasing stability, but the cases in which the one functions as an active extensor of the knee, or the other as an active dorsiflexor of the foot, must be very rare.

Lower Extremity. For quadriceps insufficiency the only operation I practise is transplantation of the tensor fascia femoris or the sartorius to the patella. Both are naturally in action in extension of the knee joint and the transference of their insertion to the patella only makes their power more effective. (Fig. I, Plate 2.)

When we come to paralysis of the foot the evidence in favour of this axiom is very striking. There are here for practical purposes only two groups of muscles - one group, the anterior tibial in action in dorsiflexion of the foot, - the other, the peroneal and posterior tibial muscles associated in action with the tendo Achillis in plantar flexion. We find that any of the anterior tibial muscles may successfully replace one another-e.g., the tibialis anticus may be transplanted to the outer side of the tarsus, or the active power in the extensor longus hallucis can be transposed to the tibialis anticus with certainty of success. On the other hand, no tendon transplanted from the peroneal or posterior tibial group will be effective in replacing the loss of active dorsiflexion. The posterior tibial and peroneal muscles are naturally associated with the tendo Achillis in extension of the ankle, so that they can be used to replace one another with certainty of success. The successful use of active posterior tibial and peroneal muscles to replace a paralysed tendo Achillis is a striking illustration of the group action of muscles in the lower extremity.

The degree of tension under which tendons should be sutured and whether their insertion should be tendon, periosteum, or bone are still matters of argument. Personally I attach much importance to suturing tendons under considerable tension and do not consider it essential even in the foot that the insertion should be into periosteum or bone.

Upper Extremity. In the upper extremity our main experience of successful tendon transplantation has been derived from war surgery where the problem was to replace the function of muscles paralysed as a result of irreparable injury to the peripheral nerves. Here we had the knowledge that the muscles utilized to replace function were physiologically normal. Although many new operations were evolved they were all essentially the application of common principles. In musculo-spiral paralysis we found that loss of power of extension of the wrist, fingers and thumb, could be surely replaced by transference of the pronator radii teres, flexor carpi radialis, and flexor carpi ulnaris to the extensors of the wrist, fingers and thumb. (Fig. II, Plate 2.)

I have also had success in transplantation of active radial and ulnar extensors of the wrist to the common and deep flexor tendons of the fingers and thumb when these muscles have been completely paralysed.

Apart from these group transplantations, success was usually more certain if we depended on one of the same group for the transplantation, e.g., in paralysis of the short extensors of the thumb. If we transfer portions of the active radial 
extensors of the wrist to these tendons, recovery of function is natural, adduction of the thumb being a movement closely and commonly associated with dorsiflexion of the wrist.

Again, paralysis of the intrinsic muscles of the hand results in loss of power of normal flexion of the metacarpo-phalangeal joints in closing the hand. Sir Harold Stiles overcame this by attaching slips of the flexor profundus tendon to the lateral border of the proximal phalanges.

It must be recognized that in many cases of paralysis the weight and leverage of the part to be controlled may be too great for the muscles available for transplantation. For this reason the opportunities for successful tendon transplantation in cases of weakness of muscles controlling the elbow, shoulder and hip, are rare. It will therefore often be found, especially in strong working men with otherwise good function in their limbs, that arthrodesis of these joints in the position of choice will give the best functional result.

\section{Tenodesis.}

The operation of utilising tendons to limit joint movement is one which also has a definite value in improvement of function-e.g., in cases of calcaneo-cavus a portion of the tendo Achillis anchored to the lower portion of the tibia will maintain a position of slight equinus where sufficient power is not present in the peroneal and posterior tibial muscles to replace the function of the tendo Achillis.

\section{(c). LESIONS OF MUSCLES AND TENDONS.}

The lesions of muscles and tendons within the scope of this paper are solutions of continuity. Such conditions as ischæmic contracture do not present any fundamental difference with regard to their treatment from that of deformities due to lower motor neurone lesions.

Solution of continuity of a muscle or tendon may be due to direct or indirect violence or to pathological changes in the tendon itself.

The work of Bunnell has placed the surgery of tendon injury on a sound basis and he rightly attaches great importance to the initial treatment, especially in cases in which the lesion is accompanied by an open wound. It cannot be too strongly stressed that the suture of cut tendons is a major surgical procedure. We must remember that cut tendons, especially flexors, tend to retract far from the site of the lesion and may carry infection with them, so that successful repair will usually depend on early elimination of sepsis and careful suture. In suturing a flexor tendon it is wise to remove the sheath at the site, in order to reduce the risk of local adhesions.

In cases of late repair of flexor tendons, where the proximal end of the tendon is much retracted, it is better to regain continuity between the flexor muscles and the terminal phalanx by means of a free transplant of the palmaris longus tendon, the proximal end being sutured to the flexor tendons at the wrist and passed subcutaneously to be anchored to the terminal phalanx.

Where complete rupture of a main tendon occurs, such as the quadriceps at its attachment to the patella or the tendo Achillis, a special problem arises because of the difficulty of overcoming their retractibility and maintaining apposition of the fragments during the period necessary for repair. Many surgeons rely upon suture by strong catgut, kangaroo tendon, or silk. My objection to the use of 
these heavy or non-absorbable sutures is not only that they tend to produce irritation and may be extruded but mainly that they cause strangulation and necrosis and so delay repair at the site of rupture.

The method I practise is to overcome muscle pull by means of a removable wire suture. This allows of accurate repair of the ruptured tendon by fine catgut. The avoidance of local reaction I feel sure expedites repair. The wire can usually be safely removed at the end of two or three weeks.
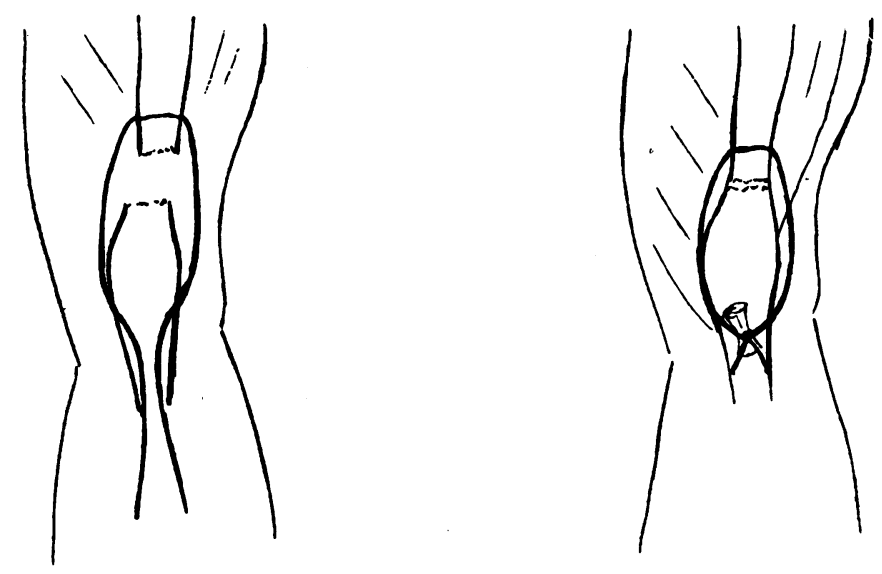

(a) Use of removable wire-suture in repair of ruptured quadriceps.
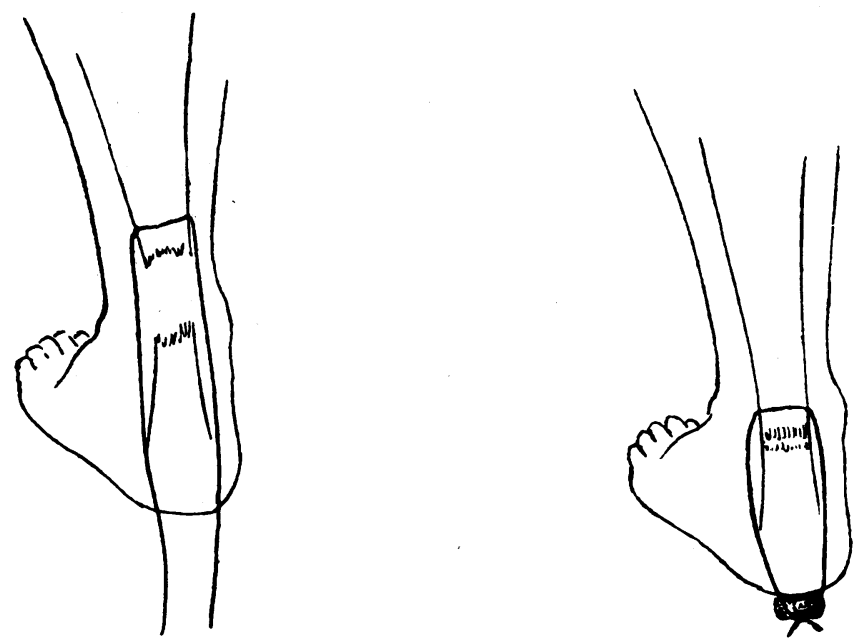

(b) Use of removable wire suture in repair of ruptured tendo Achillis.

Fig. 3.-Showing method of temporary wire suture. 
I use a flexible phosphor bronze wire. After exposure of the site of rupture it is passed sinuously in a transverse direction through the proximal fragment. In the case of rupture of the quadriceps tendon the ends threaded on separate needles are then passed along the lateral border of the patella to emerge through the skin just above the tuberosity of the tibia. They are then drawn sufficiently tight to completely relax the quadriceps muscle and tied over a pad of gauze.

In some cases it may be necessary to pass one of the wires through the tuberosity of the tibia in order to obtain a more secure purchase.

In cases of high rupture of the tendo Achillis the procedure is similar, the wires emerging about $\frac{1}{4}$ in. apart on the posterior border of the heel.

In cases of rupture of the tendo Achillis in which the rupture is really an evulsion of the tendon from the muscle, the line of suture and approximation of the fragments may be reinforced by transplantation of the peroneus longus tendon to the os calcis. The tendon is divided below the external malleolus, drawn into the posterior wound, and passed several times through the proximal and distal fragments before being sutured into the posterior aspect of the os calcis.

Available space has allowed of only a general survey of the principles which I believe should guide us in approaching the problem of the surgical treatment of muscles and tendons in relation to paralysis and injury. The points which I have emphasized are:-

(I) That in lesions of the upper motor neurone-deformity is due to muscle spasm, so that division of muscles and tendons is usually a necessary preliminary to restoration of the best position of the joints for function.

(2) That in lesions of the lower motor neurone-as muscle power is already deficient every effort should be made to correct deformity by gradual methods, rather than by division of tendons.

(3) That arthrodesis or tenodesis may be an essential preliminary to tendon transplantation to give the best final functional result.

(4) That success in tendon transplantation depends largely on the selection of tendons and the tension under which they are sutured.

(5) That in certain tendon ruptures removable wire sutures should be used to maintain relaxation of powerful muscles. 